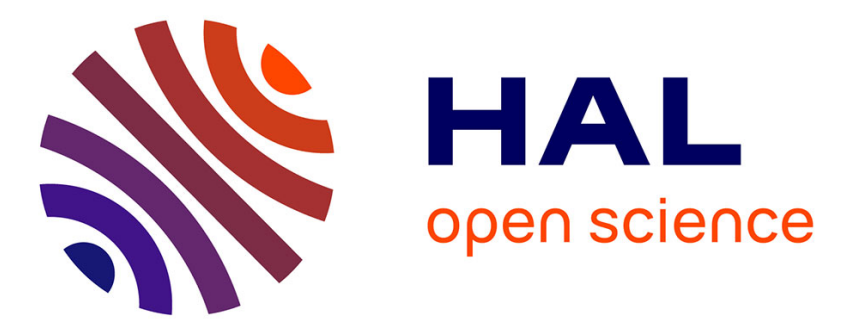

\title{
Multi-packet Hybrid ARQ: Closing gap to the ergodic capacity
}

Mohammed Jabi, Aata El Hamss, Leszek Szczecinski, Pablo Piantanida

\section{To cite this version:}

Mohammed Jabi, Aata El Hamss, Leszek Szczecinski, Pablo Piantanida. Multi-packet Hybrid ARQ: Closing gap to the ergodic capacity. IEEE Transactions on Communications, 2015, 10.1109/TCOMM.2015.2493138 . hal-01257567

\section{HAL Id: hal-01257567 https://hal.science/hal-01257567}

Submitted on 16 Sep 2020

HAL is a multi-disciplinary open access archive for the deposit and dissemination of scientific research documents, whether they are published or not. The documents may come from teaching and research institutions in France or abroad, or from public or private research centers.
L'archive ouverte pluridisciplinaire HAL, est destinée au dépôt et à la diffusion de documents scientifiques de niveau recherche, publiés ou non, émanant des établissements d'enseignement et de recherche français ou étrangers, des laboratoires publics ou privés. 


\title{
Multi-packet Hybrid ARQ: Closing gap to the ergodic capacity
}

\author{
Mohammed Jabi, Aata El Hamss, Leszek Szczecinski, and Pablo Piantanida
}

\begin{abstract}
In this work we consider incremental redundancy (IR) hybrid automatic repeat request (HARQ), where transmission rounds are carried out over independent block-fading channels. We propose the so-called multi-packet HARQ where the transmitter allows different packets to share the same channel block. In this way the resources (block) are optimally assigned throughout the transmission rounds. This stands in contrast with the conventional HARQ, where each transmission round occupies the entire block. We analyze superposition coding and time-sharing transmission strategies and we optimize the parameters to maximize the throughput. Besides the conventional one-bit feedback (ACK/NACK) we also consider the rich, multi-bit feedback. To solve the optimization problem we formulate it as a Markov decision process (MDP) problem where the decisions are taken using accumulated mutual information (AMI) obtained from the receiver via delayed feedback. When only one-bit feedback is used to inform the transmitter about the decoding success/failure (ACK/NACK), the Partial State Information Markov Decision Process (PSI-MDP) framework is used to obtain the optimal policies. Numerical examples obtained in a Rayleigh-fading channel indicate that, the proposed multi-packet HARQ outperforms the conventional one, by more than $5 \mathrm{~dB}$ for high spectral efficiencies.
\end{abstract}

\section{Index Terms}

Block Fading Channels, Hybrid Automatic Repeat reQuest, Partial State Information, Markov Decision Process, Superposition Coding, Time Sharing.

M. Jabi and L. Szczecinski are with INRS-EMT, Montreal, Canada. [e-mail: \{jabi, leszek\}@emt.inrs.ca].

A. El Hamss is with InterDigital Communications, Montreal, Canada. [e-mail: aata.elhamss@gmail.com].

P. Piantanida is with SUPELEC, Gif-sur-Yvette, France. [e-mail: pablo.piantanida@supelec.fr].

Part of this work will be presented at the IEEE Global Communication Conference (GLOBECOM), Austin, Texas, USA, December 2014.

The work was supported by the government of Quebec, under grant \#PSR-SIIRI-435. 


\section{INTRODUCTION}

I

$\mathrm{N}$ THIS work we propose and optimize the strategies to increase the throughput of HARQ transmission over a block fading channel.

HARQ is mostly used to deal with the loss of data packets due to unpredictable changes in the channel and consists in "handshaking" between the transmitter and the receiver: the receiver sends the binary messages via a feedback channel to inform the transmitter about the success (ACK message) or failure (NACK) of the transmission. Then a new version of the lost packet is transmitted. This process continues till ACK is received or-in the case of truncated HARQ- till the maximum number of transmission rounds is reached. In practice, the truncation appears as an implementation constraint but also may be justified when transmitting data that is delay-sensitive, which after a prescribed time may loose its validity.

HARQ is often perceived as an additional "guarantee", which works on the top of the physical layer (PHY). However, it was already shown in previous works, that adjusting the PHY-related parameters (rate, coding, power) as a function of the HARQ state can significantly improve the performance, e.g., in terms of the average transmission rate (throughput) [1]-[5], or the outage [6]-[8].

While the parameters of the PHY may be adjusted as a function of the number of received NACK messages, more substantial gains are obtained exploiting the additional information sent by the receiver. We thus consider the case when, on top of ACK/NACK messages, the receiver conveys over the feedback channel, "multi-bit" receiver state information (RSI). As we explain later, RSI represent the state of the decoder, which is related to the channel state information (CSI) determined by the channel gains in different blocks. These channel gains are assumed to be independent and identically distributed (i.i.d.) random variables (a modelling, which captures adequately the fact that retransmissions are usually scheduled in well-spaced time instant to absorb the processing and round-trip delays). Therefore, the transmitter is unable to infer the instantaneous CSI from the RSI, and thus, the conventional adaptive modulation and coding (AMC) cannot be used.

Nevertheless, the RSI that accompanies the NACK message, provides the transmitter with valuable prior allowing it to suitably adjust its PHY parameters in the subsequent rounds. Indeed, the use of RSI was already considered before in [3], [5], [9] to adapt the length of the codewords 
with the implicit objective of shortening the average number of transmissions and using the time "released" in such a way to send more packets.

It is worth mentioning here that, in [3], [5], [9] the lengths were optimized in abstraction of the system-level considerations. Thus, exploiting the released time is not obvious because the codewords with variable lengths are sent within the same block. The lengths are optimized in abstraction of the block-length so, in general, they do not match the latter. This, will produce an unoccupied time within the block, which in turn translates into a throughput loss. To address this problem, [5], [10] proposed the use of many packets within a single block, reducing in this way the impact of the unoccupied time. This may go, however, against the practical considerations of having only one or a few packets in the block. Moreover, regardless of systemlevel considerations, recognizing that various packets are transmitted within the same block raises the question of optimality of the conventional time-sharing (TS) approach, and the superposition coding (SC) [11] may be an alternative as already considered before with HARQ, e.g., in [12][14].

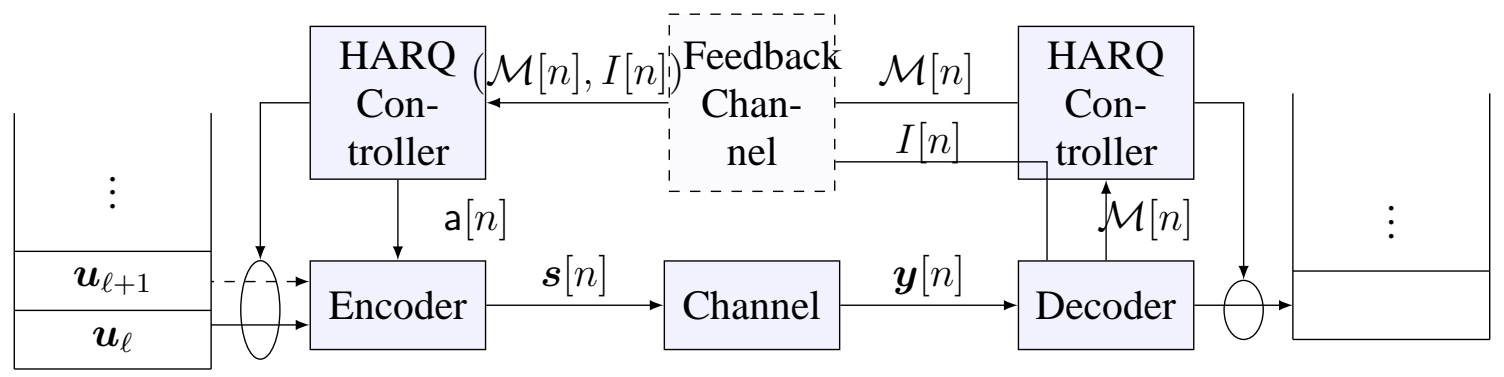

Fig. 1: Model of the HARQ transmission: the HARQ controller uses the information obtained over the feedback channel to take actions a $[n]$, which determine the encoding.

In this work we address these two issues. On one hand, we explicitly consider the constraints resulting from the transmission of variable-length packets within the same block [15]; this links the HARQ design with system-level considerations, an issue that was lacking in [3], [5]. On the other hand, we consider the SC and TS as alternatives for the joint encoding of the new and retransmitted packets; we formally optimize the parameters which was not considered in [12]-[14].

We look at the HARQ as a control process based on the feedback signal and we optimize the actions which are given by the joint encoding (and its parameters) to be used. To solve the 
optimization we define our problem as a Markov decision process (MDP) [16, Ch. 7.4], which was already used in different contexts for HARQ optimization, e.g., in [17], [18]. We maximize the throughput which is a relevant performance criterion as it can be directly related to the ergodic (long term) channel capacity [19], [20].

The paper is organized as follows: Sec. II defines the model of the system under study, which is then cast into MDP in Sec. III-A. We explain the optimization in Sec. III-B and the numerical results are shown and discussed in Sec. III-C. The case of one bit feedback is analyzed in Sec. IV. We conclude the work in Sec. V.

\section{MOdel OF HARQ}

We first describe the conventional HARQ, which allows us to define the useful notation and next we discuss the multi-packet HARQ we propose.

\section{A. Conventional HARQ and Notations}

We consider a point-to-point transmission using HARQ shown in Fig. 1, where the transmitter sends the data block $s[n]$ of $N_{\mathrm{s}}$ symbols over a block-fading channel. Each block $n$ contains the encoded version of $N_{\mathrm{b}}$ information bits contained in $\boldsymbol{u}_{\ell}$, where $\ell$ indicates the head of the line (HoL) packet at block time $n$ (the HoL packet is the first packet in the buffer to be transmitted); for convenience we say that the packet $\boldsymbol{u}_{\ell+1}$ is "HoL-next". The coding rate per packet is thus $R=N_{\mathrm{b}} / N_{\mathrm{s}}$. The receiver observes the channel outcome $\boldsymbol{y}[n]$ and attempts to decode $\boldsymbol{u}_{\ell}$. We note that the indices of the packets are not the same as indices of the blocks, we refer to the former via subindexing, e.g., $\boldsymbol{u}_{\ell}$, and-to the latter-via arguments within brackets, e.g., $\boldsymbol{s}[n]$. We also use $n_{\ell}$ to denote the index of the block $s[n]$ when the packet $\boldsymbol{u}_{\ell}$ was transmitted for the first time.

More than one transmission may be necessary to deliver the packet and the index of the transmission round of the HoL packet is kept by transmitter in the HARQ counter $k_{\ell}$. For convenience, we assume that the HARQ counter $k$ for the $t$-th packet entering the transmitter's buffer, where $t>\ell$ is set to zero, i.e., $k_{t} \leftarrow 0$. The decoding errors are detected at the receiver which sends to the transmitter a binary message $\mathcal{M}[n]=\mathcal{M}_{\ell, k_{\ell}}$, where $\mathcal{M}_{\ell, k_{\ell}}=$ ACK if the decoding of $\boldsymbol{u}_{\ell}$ is successful in the $k_{\ell}$-th round, or $\mathcal{M}_{\ell, k_{\ell}}=$ NACK if the decoding fails. Reassume that the feedback channel is error-free. 
Due to the propagation, transmission, and processing delays, the message $\mathcal{M}[n]$ arrives at the transmitter at time block $n+1$, where it can be used by the HARQ controller. The latter discards the HoL packet when ACK is received or the maximum number of transmission rounds $K$ is reached; then the HoL-next packet becomes the HoL. Formally, this is done, first, incrementing the HoL index, which otherwise does not change, i.e.,

$$
\ell \leftarrow \begin{cases}\ell+1, & \text { if } \mathcal{M}_{\ell, k_{\ell}}=\mathrm{ACK} \text { or } k_{\ell}=K \\ \ell, & \text { otherwise }\end{cases}
$$

and, next, increasing the HARQ counter of the HoL packet

$$
k_{\ell} \leftarrow k_{\ell}+1
$$

When the packets have their counter sets to zero, increasing it via (2), we obtain $k_{\ell}=1$, which means that we start the HARQ process of the packet $\boldsymbol{u}_{\ell}$.

The encoding, in general depend on the index of the transmission round, i.e., on the HARQ counter $k_{\ell}$, i.e.,

$$
\boldsymbol{s}[n]=\Phi_{k_{\ell}}\left(\boldsymbol{u}_{\ell}\right) .
$$

In particular, when $\Phi_{k}\left(\boldsymbol{u}_{\ell}\right)=\Phi_{1}\left(\boldsymbol{u}_{\ell}\right), k=1, \ldots, K$ we have the case of transmission with the repetition redundancy (RR) [21] [22]. That is, irrespectively of $k_{\ell}$, the transmitted symbols $\boldsymbol{s}[n]$ are always the same for the given $\boldsymbol{u}_{\ell}$. If, on the other hand, $k_{\ell}$ is used to extract different subcodewords of the mother code's codeword

$$
\left[\Phi_{1}(\boldsymbol{u}), \ldots, \Phi_{K}(\boldsymbol{u})\right],
$$

we obtain the well-known incremental redundancy HARQ (HARQ-IR) [23], where conventionally, all the subcodewords $\Phi_{k_{\ell}}$ have the same length. HARQ-IR is the focus of this work.

The channel outcome at the receiver is given by

$$
\boldsymbol{y}[n]=\sqrt{\operatorname{snr}[n]} \boldsymbol{s}[n]+\boldsymbol{z}[n],
$$

where $\operatorname{snr}[n]$ is the channel signal-to-noise ratio (SNR), $z[n]$ is a zero mean, unitary-variance Gaussian variable modelling noise. We assume that $\operatorname{snr}[n]$ are i.i.d. and their probability density 
function (PDF) is known. For numerical evaluation we use the exponential form (i.e., we consider Rayleigh block fading)

$$
p_{\mathrm{snr}}(x)=\overline{\mathrm{snr}}^{-1} \exp (-x / \overline{\mathrm{snr}})
$$

where $\overline{\mathrm{snr}}$ is the average SNR.

In HARQ-IR, the receiver decodes the packet $\boldsymbol{u}_{\ell}$ concatenating the $k \leq K$ blocks of channel outcomes

$$
\boldsymbol{y}_{\ell, k} \triangleq\left[\boldsymbol{y}\left[n_{\ell}\right], \ldots, \boldsymbol{y}\left[n_{\ell}+k-1\right]\right]
$$

Then, assuming the subcodewords $\Phi_{k}\left(\boldsymbol{u}_{\ell}\right)$ are drawn from randomly generated codebook, and for sufficiently large $N_{\mathrm{s}}$, the decoding is successful if the accumulated mutual information (AMI) defined as

$$
I_{\ell, k} \triangleq \sum_{t=0}^{k-1} C\left(\operatorname{snr}\left[n_{\ell}+t\right]\right)
$$

exceeds the transmission rate, i.e.,

$$
I_{\ell, k}>R
$$

For simplicity, we assume only Gaussian inputs, i.e.,

$$
C(\mathrm{snr}) \triangleq \log _{2}(1+\mathrm{snr})
$$

The AMI is thus equivalent to the RSI and we may sent it to the transmitter over the feedback channel but it is irrelevant for the conventional HARQ.

\section{B. Multi-packet $H A R Q$}

In the conventional HARQ, $s[n]$ depends solely on the HARQ counter $k_{\ell}$ and the packet contents $\boldsymbol{u}_{\ell}$, as per (3). For the proposed multi-packet HARQ, we assume that the encoder is able to jointly encode the HoL packet $\boldsymbol{u}_{\ell}$ and the HoL-next packet $\boldsymbol{u}_{\ell+1}$ as shown also schematically in Fig. 1, i.e.,

$$
\boldsymbol{s}[n]=\Phi^{\mathrm{MP}}\left(\boldsymbol{u}_{\ell}, \boldsymbol{u}_{\ell+1}, k_{\ell}, k_{\ell+1}, p\right),
$$


where $p$ is the parameter of the encoding and it is a function of the counters $k_{\ell}$ and $k_{\ell+1}$ and the RSI.

We consider four possible encoding modes

- Conventional, one-packet transmission, which we denote by '1P', where the block $s[n]$ is occupied only by the subcodewords of the HoL packet,

- Dropped, one-packet transmission, which we denote by '0P', where we stop the transmission of the HoL packet and $s[n]$ is occupied only by the subcodewords of the HoL-next packet,

- TS transmission, where the subcodewords of the HoL and HoL-next packets are transmitted in non-overlaping parts of the block, with the time-sharing defined by $p$, and

- SC transmission, where both codewords are superimposed with power fractions defined by $p$.

We clarify this in Table I, where we add details to the encoding notation in (3) as follows:

$$
\Phi_{k_{\ell}}\left(\boldsymbol{u}_{\ell}, \alpha\right)
$$

where $\alpha$ indicates the relative length of the $k$ th subcodeword composed of $\alpha N_{\mathrm{s}}$ symbols.

\begin{tabular}{l|c}
$\mathrm{a}[n]$ & $\Phi^{\mathrm{MP}}\left(\boldsymbol{u}_{\ell}, \boldsymbol{u}_{\ell+1}, k_{\ell}, k_{\ell+1}, p\right)$ \\
\hline \hline$(1 \mathrm{P},-)$ & $\Phi_{k_{\ell}}\left(\boldsymbol{u}_{\ell}, 1\right)$ \\
\hline$(0 \mathrm{P},-)$ & $\Phi_{k_{\ell+1}}\left(\boldsymbol{u}_{\ell+1}, 1\right)$ \\
\hline$(\mathrm{TS}, p)$ & {$\left[\Phi_{k_{\ell}}\left(\boldsymbol{u}_{\ell}, p\right), \Phi_{k_{\ell}+1}\left(\boldsymbol{u}_{\ell+1}, 1-p\right)\right]$} \\
\hline$(\mathrm{SC}, p)$ & $\sqrt{p} \Phi_{k_{\ell}}\left(\boldsymbol{u}_{\ell}, 1\right)+\sqrt{1-p} \Phi_{k_{\ell+1}}\left(\boldsymbol{u}_{\ell+1}, 1\right)$
\end{tabular}

TABLE I: Results of encoding actions $\mathrm{a}[n]$ for the joint encoding of the HoL and HoL-next packets $\boldsymbol{u}_{\ell}$ and $\boldsymbol{u}_{\ell+1}$.

We note that when the joint encoding (TS or SC) is chosen, the feedback channel transmits the decoding result for both HoL and HoL-next packets, i.e., $\mathcal{M}[n]=\left(\mathcal{M}_{\ell, k_{\ell}}, \mathcal{M}_{\ell+1, k_{\ell+1}}\right)$.

The role of the HARQ controller is to decide on the encoding actions $\mathrm{a}[n]=(\mathrm{m}[n], p[n])$, where $\mathrm{m}[n] \in \mathcal{A}_{\text {mod }}=\{1 \mathrm{P}, 0 \mathrm{P}, \mathrm{TS}, \mathrm{SC}\}$ defines the encoding "mode" and $\left.p[n] \in \mathcal{A}_{\mathrm{p}}=\right] 0,1[$ is the encoding parameter as specified also in Table I. The conventional HARQ always takes the same encoding action $\mathrm{a}[n]=(1 \mathrm{P},-)$, where we use "-" to indicate that, in this case, the parameter $p$ is irrelevant from the point of view of the encoding. The actions are taken from the action-space $\mathcal{A}=\mathcal{A}_{\text {mod }} \times \mathcal{A}_{\mathrm{p}}$ and may result in one-packet (1P or 0P) or a multi-packet (SC or 
TS) transmissions.

The rule for updating $\ell$ becomes now the following:

$$
\ell \leftarrow \begin{cases}\ell+1, & \text { if } \mathcal{M}_{\ell, k_{\ell}}=\mathrm{ACK} \text { or } k_{\ell}=K \text { or } \mathrm{m}=0 \mathrm{P} \\ \ell, & \text { otherwise }\end{cases}
$$

and the HARQ counters' update takes into account the possibility of joint encoding

$$
\begin{aligned}
& k_{\ell} \leftarrow k_{\ell}+1, \forall \mathrm{m} \in \mathcal{A}_{\text {mod }}, \\
& k_{\ell+1} \leftarrow\left\{\begin{array}{ll}
0, & \text { if } \mathrm{m} \in\{1 \mathrm{P} \cup 0 \mathrm{P}\} \\
k_{\ell+1}+1, & \text { if } \mathrm{m} \in\{\mathrm{TS} \cup \mathrm{SC}\}
\end{array},\right.
\end{aligned}
$$

where the second condition in (15) reflects the fact that both, HoL and HoL-next packets are transmitted simultaneously.

We note that the actions from the subspace $\mathcal{A}_{\text {mod }} \times\{0,1\}$ are explicitly excluded because for the joint encoding with $p \in\{0,1\}$, one of the packets is deprived of the transmission time (TS) or power (SC), while its HARQ counter would increment as per the second line of (15). This is clearly suboptimal so the cases of $p \in\{0,1\}$ are handled by the encoding modes $1 \mathrm{P}$ or $0 \mathrm{P}$, where only one HARQ counter is incremented.

We also note that the action $(0 \mathrm{P},-)$ was already used in [5] [24] and means that the packet is abandoned when there is no "reasonable hope" to decode it successfully. While making such a decision may seem drastic, the dropped packeted may be re-injected into the transmitter's buffer as may also be other packets considered lost. This issue depends on the sensitivity of the source to the delay in the packets' delivery-the problem we do not consider here.

The decoding is done in the similar way as in the one-packet HARQ. In the case of the TS encoding, after the $k$-th transmission, the decoding is successful provided that $I_{\ell, k}>R$, with

$$
I_{\ell, k}=I_{\ell, k-1}+p C(\mathrm{snr})
$$

where by definition $I_{\ell, 0}=0$ and to simplify the notation we used snr $\equiv \operatorname{snr}\left[n_{\ell}+k-1\right]$. 
At the same time we find the AMI for the HoL-next packet as

$$
I_{\ell+1, k}=I_{\ell+1, k-1}+(1-p) C(\mathrm{snr}) .
$$

Since snr is random, increasing $p$, the probability of successful decoding of the packet $\boldsymbol{u}_{\ell}$ increases with $p$, see (16), but at the same time, the probability of correct decoding of the HoLnext packet is decreased. Thus, it is not possible to improve simultaneously the reliability of transmission of both packets. The challenge of optimizing the encoding actions lies in striking the balance between these contradictory effects.

In the case of the SC, the decoding is slightly more involved because the HoL and HoL-next packets interfere with each other. For simplicity we only consider single packet decoding, in contrast to joint decoding of both packets ${ }^{1}$. That is, we assume that decoding of $\boldsymbol{u}_{\ell}$ depends solely on $I_{\ell, k-1}$ and $\boldsymbol{y}\left[n_{\ell}+k-1\right]$, i.e., the AMI of the HoL packet is given by

$$
I_{\ell, k}=I_{\ell, k-1}+C\left(\frac{p s n r}{1+(1-p) \mathrm{snr}}\right)
$$

The AMI for the HoL-next packet is given by

$$
I_{\ell+1, k}=\left\{\begin{array}{llc}
I_{\ell+1, k-1}+C((1-p) \mathrm{snr}) & \text { if } \quad & I_{\ell, k}>R \\
I_{\ell+1, k-1}+C\left(\frac{(1-p) \mathrm{snr}}{1+p \mathrm{snr}}\right) & \text { if } & I_{\ell, k} \leq R
\end{array}\right.
$$

in the case $\left\{I_{\ell, k}>R\right\}$ we assume that the interference induced by the superposed subcodeword $\Phi_{k_{\ell}}\left(\boldsymbol{u}_{\ell}\right)$ was removed; in the other case-that the interference cannot be removed because the AMI related to the HoL packet is not sufficiently large to allow for decoding $\left(I_{\ell, k} \leq R\right)$.

\section{MULTI-BIT FEEDBACK}

We assume that after each multi-packet HARQ round, on top of the conventional signalling $\mathcal{M}[n]=\left(\mathcal{M}_{\ell, k_{\ell}}, \mathcal{M}_{\ell+1, k_{\ell+1}}\right)$, the transmitter is provided with additional information about the state of the receiver. In particular, since the decoding success/failure are determined by the AMI, see (9), the AMI $\boldsymbol{I}[n]=\left(I_{\ell, k_{\ell}}, I_{\ell+1, k_{\ell+1}}\right)$ is sent via the feedback channel to the transmitter ${ }^{2}$, as shown in Fig. 1. We focus on optimizing the encoding action a, targeting the maximization of

\footnotetext{
${ }^{1}$ While joint decoding is possible (as we transformed our point-to-point channel into multiple access channel), joint decoding would result in an additional complex at the receiver and on the other hand the analysis would become much involved.

${ }^{2}$ We may send $I_{\ell, k_{\ell}}$ or, instead, report $\operatorname{snr}[n]$ and let the transmitter to calculate the AMI via (16), (17), (18), or (19)
} 
the throughput. The key idea is to represent the multi-packet HARQ as an MDP $(\mathcal{S}, \mathcal{A}, \mathcal{W}, Q, r)$ where $\mathcal{S}, \mathcal{A}$ and $\mathcal{W}$ are the state space, the action space and the disturbance space respectively, $Q$ is the transition law and $r$ is the reward [16, Ch. 1].

Being in state $\mathrm{s} \in \mathcal{S}$ at block time $n$ gives the controller the possibility to take action a $[n] \in$ $\mathcal{A}(\mathrm{s})$, after which the HARQ moves to the next state $\mathrm{s}^{\prime} \in \mathcal{S}$ at time $n+1$. In general, not all actions are allowed in a state $\mathrm{s}$, thus $\bigcup_{\mathrm{s} \in \mathcal{S}} \mathcal{A}(\mathrm{s})=\mathcal{A}$. The transition from the state $\mathrm{s}$ to $\mathrm{s}^{\prime} \in \mathcal{S}$ depends uniquely on action $\mathrm{a}[n]$ and on a random disturbance $\mathrm{w}[n] \in \mathcal{W}$. Thus, the transition between states is characterized in probabilistic manner described by the transition law $Q$. The function $r(\mathrm{~s}, \mathrm{a}): \mathcal{S} \times \mathcal{A} \rightarrow \mathbb{R}$ defines the reward acquired when the system is in state $\mathrm{s}$ and the controller chooses action a.

\section{A. Markov Decision Process}

In the multi-packet HARQ, the feedback messages AMI $\boldsymbol{I}[n]$, as well as the HARQ counters $\boldsymbol{k}[n]=\left(k_{\ell}, k_{\ell+1}\right)$ define the state of the HARQ process. The AMI $\boldsymbol{I}$ is defined over the set $\mathcal{I}^{2}$ by the quantities:

$$
\begin{gathered}
\mathcal{I} \triangleq[0, R] \cup \mathcal{I}_{R_{+}}, \\
\mathcal{I}_{R_{+}} \triangleq(R, \infty),
\end{gathered}
$$

where (21) explicitly groups those values of the AMI which lead to the event of decoding success $\left(I_{\ell, k}>R\right)$. With $K$ allowed transmission rounds and considering that HoL and HoLnext packets may be sent simultaneously, the HARQ counters $\boldsymbol{k}$ can only take values in the set $\mathcal{K}=\{(1,0),(2,1),(2,0), \ldots,(K, K-1),(K, K-2), \ldots,(K, 0)\}$. Thus, the state space $\mathcal{S}$ is defined as: $\mathcal{S}=\mathcal{K} \times \mathcal{I}^{2}$. Each state is thus represented by a quadruplet $\left(k_{\ell}, k_{\ell+1}, I_{\ell}, I_{\ell+1}\right)$. We note that the value of AMI $\boldsymbol{I}[n]$ contains implicitly the results of the decoding $\mathcal{M}[n]$.

For $\mathrm{s} \in \mathcal{S}$, we denote by $\mathcal{M}^{\mathrm{s}}, \boldsymbol{k}^{\mathrm{s}}$ and $\boldsymbol{I}^{\mathrm{s}}$ the corresponding value of $\mathcal{M}, \boldsymbol{k}$ and $\boldsymbol{I}$ respectively. 
We distinguish the following subsets:

$$
\begin{gathered}
\mathcal{S}_{\mathrm{ACK}, \mathrm{ACK}}=\left\{\mathrm{s} \in \mathcal{S} \mid \mathcal{M}^{\mathrm{s}}=(\mathrm{ACK}, \mathrm{ACK})\right\} \\
\mathcal{S}_{\mathrm{NACK}, \mathrm{NACK}}=\left\{\mathrm{s} \in \mathcal{S} \mid \mathcal{M}^{\mathrm{s}}=(\mathrm{NACK}, \mathrm{NACK})\right\} \\
\mathcal{S}_{\mathrm{ACK}, \mathrm{NACK}}=\left\{\mathrm{s} \in \mathcal{S} \mid \mathcal{M}^{\mathrm{s}} \in\{(\mathrm{ACK}, \mathrm{NACK}),\right. \\
(\mathrm{NACK}, \mathrm{ACK})\}\} \\
\mathcal{S}_{\mathrm{ACK}}^{1 \mathrm{P}}=\left\{\mathrm{s} \in \mathcal{S} \mid\left\{\mathcal{M}^{\mathrm{s}}=(\mathrm{ACK},-)\right.\right. \\
\left.\left.\cap \boldsymbol{k}^{\mathrm{s}}=(l, 0), 1 \leq l \leq K\right\}\right\} \\
\mathcal{S}_{\mathrm{NACK}}^{1 \mathrm{P}}=\left\{\mathrm{s} \in \mathcal{S} \mid\left\{\mathcal{M}^{\mathrm{s}}=(\mathrm{NACK},-)\right.\right. \\
\left.\left.\cap \boldsymbol{k}^{\mathrm{s}}=(l, 0), 1 \leq l \leq K\right\}\right\}
\end{gathered}
$$

where $\mathcal{S}_{\mathrm{ACK}, \mathrm{ACK}}$ contains states when both packets are decoded correctly while $\mathcal{S}_{\mathrm{NACK}, \mathrm{NACK}}$ presents states when both packets failed to be decoded, $\mathcal{S}_{\mathrm{ACK}, \mathrm{NACK}}$ is the set of states when only one packet is decoded correctly, $\mathcal{S}_{\mathrm{ACK}}^{1 \mathrm{P}}$ and $\mathcal{S}_{\mathrm{NACK}}^{1 \mathrm{P}}$ characterize the states when the one-packet transmission mode is adopted in the whole HARQ rounds and that the packet is successfully or unsuccessfully decoded respectively.

We also consider three types of HARQ differentiated by their respective action space:

1) Conventional one-packet HARQ (1P) when $p$ is irrelevant. In this case the action space $\mathcal{A}$ has only one element $(1 \mathrm{P},-)$ and no optimization is needed.

2) Time sharing multi-packet mode (TS) when the action space is defined as $\mathcal{A}=\mathcal{A}_{\text {mod }}^{\mathrm{TS}} \times \mathcal{A}_{\mathrm{p}}$ with $\mathcal{A}_{\text {mod }}^{\mathrm{TS}}=\{1 \mathrm{P}, 0 \mathrm{P}, \mathrm{TS}\}$.

3) Superposition coding multi-packet mode (SC) when $\mathcal{A}=\mathcal{A}_{\bmod }^{\mathrm{SC}} \times \mathcal{A}_{\mathrm{p}}$ with $\mathcal{A}_{\text {mod }}^{\mathrm{SC}}=\{1 \mathrm{P}, 0 \mathrm{P}, \mathrm{SC}\}$.

We assume that the joint-encoding actions, i.e., $\mathrm{m} \in\{\mathrm{TS}, \mathrm{SC}\}$, can be taken for all states $\mathrm{s} \in \mathcal{S}$ except if $\mathrm{s} \in\left\{\mathcal{S}_{\mathrm{ACK}, \mathrm{ACK}} \cup \mathcal{S}_{\mathrm{ACK}}^{\mathrm{IP}}\right\}$ when only one packet is transmitted. That is, the joint-encoding actions is only allowed when the HoL packet is not succesfully decoded.

The statistically evolution of the system is represented by the transition law $Q$. Since the states and the actions are discrete, see Sec. III-B1, this law is given by the state-to-state transition probabilities:

$$
p_{\mathrm{s}, \mathrm{s}^{\prime}}(\mathrm{a}) \triangleq \operatorname{Pr}\left\{\mathrm{s}[n+1]=\mathrm{s}^{\prime} \mid \mathrm{s}[n]=\mathrm{s}, \mathrm{a}[n]=\mathrm{a}\right\}
$$


which can be calculated using (16)-(19). An example of $p_{\mathrm{s}, \mathrm{s}^{\prime}}$ calculation is shown in Appendix A. We assume here the stationary behaviour, in which the time $n$ is irrelevant for the transition probability; this condition is satisfied for sufficiently large $n$, i.e., after the transients phase.

The transition from the state $\mathrm{s}[n]$ to the state $\mathrm{s}[n+1]$ depends on the action $\mathrm{a}[n]$ and on the disturbance $\operatorname{snr}[n] \geq 0$ as can be seen in (16)-(19). Consequently we take $\mathcal{W}=\mathbb{R}^{+}$.

Each state-transition yields the reward given by

$$
\hat{r}\left(\mathrm{~s}, \mathrm{a}, \mathrm{s}^{\prime}\right)= \begin{cases}R, & \mathrm{~s}^{\prime} \in\left\{\mathcal{S}_{\mathrm{ACK}, \mathrm{NACK}}, \mathcal{S}_{\mathrm{NACK}, \mathrm{ACK}}, \mathcal{S}_{\mathrm{ACK}}^{1 \mathrm{P}}\right\} \\ 2 R, & \mathrm{~s}^{\prime} \in \mathcal{S}_{\mathrm{ACK}, \mathrm{ACK}} \\ 0, & \text { otherwise }\end{cases}
$$

and the expected reward for taking action a in the state $\mathrm{s}$ is then given by

$$
r(\mathrm{~s}, \mathrm{a})=\sum_{\mathrm{s}^{\prime} \in \mathcal{S}} p_{\mathrm{s}, \mathrm{s}^{\prime}}(\mathrm{a}) \hat{r}\left(\mathrm{~s}, \mathrm{a}, \mathrm{s}^{\prime}\right) .
$$

Our objective is thus to find a policy $\pi: \mathcal{S} \mapsto \mathcal{A}$, such that the HARQ controller taking actions $\mathrm{a}=\pi(\mathrm{s}) \in \mathcal{A}$, maximizes the throughput defined as

$$
\eta(\pi)=\lim _{N \rightarrow \infty} \frac{1}{N} \sum_{n=1}^{N} \mathbb{E}[r(\mathrm{~s}[n], \pi(\mathrm{s}[n])),],
$$

where the expectation is taken with respect to the random states $s[1], \ldots, s[N]$ of the HARQ process, and $r(\mathrm{~s}[n], \pi(\mathrm{s}[n]))$ is the random reward obtained using the actions $\pi(\mathrm{s}[n])$ after transmission of the block $n$ defined in (29).

\section{B. Throughput optimization}

1) Discretization: The problem is now formulated as the MDP and, in order to make it tractable numerically we make the state-space $\mathcal{S}$ discrete. The first dimension $\mathcal{K}$ of $\mathcal{S}$ is discrete by definition, so we discretize $\mathcal{I}$ using $T_{\mathcal{I}}$ points; which means that we need to discretize the set $\left[0, R\left[\right.\right.$ using $T_{\mathcal{I}}-1$ points (we used uniform quantization) and assign one discretization point to $\mathcal{I}_{R_{+}}$.

Similarly, the actions set $\mathcal{A}$ is discretized over $T_{\mathrm{p}}$, thus the encoding parameters $p \in \mathcal{A}_{\mathrm{p}}$ are discretized over $T_{\mathrm{p}}-2$ points. 
2) Policy optimization: Our goal is to solve the following optimization problem

$$
\eta^{*}=\max _{\pi \in \Pi} \eta(\pi)
$$

where $\Pi$ is the set of admissible policies $\pi: \mathcal{S} \mapsto \mathcal{A}$.

This average reward-per-stage problem can be solved using the so-called Bellman's equations [16, Ch. 7.4],

$$
\begin{aligned}
\eta^{*}+h_{\mathrm{s}} & =\max _{a \in \mathcal{A}(\mathrm{s})}\left[r(\mathrm{~s}, \mathrm{a})+\sum_{\mathrm{s}^{\prime} \in \mathcal{S}} p_{\mathrm{s}, \mathrm{s}^{\prime}}(\mathrm{a}) h_{\mathrm{s}^{\prime}}\right], \quad \forall \mathrm{s} \neq \mathrm{s}_{\mathrm{p}}, \\
h_{\mathrm{sp}_{\mathrm{p}}} & =0
\end{aligned}
$$

thanks to the following lemma.

Lemma 1. There is at least one state, denoted by $s_{p}$ and $m>0$, such that, for all initial states and all policies $\pi$, the probability of being in state $\mathrm{s}_{p}$ at least once within the first $m$ times, is non zero, i.e., $\operatorname{Pr}\left\{\mathrm{s}[n]=\mathrm{s}_{p}\right\}>0$, where $n<m$.

Proof: We take the special state $\mathrm{s}_{\mathrm{p}}=\left(1,0, I_{\mathrm{p}}, 0\right)$, where $I_{\mathrm{p}}$ is the smallest value defined by the discretization. Since NACK message occurs with non-zero probability, and the probability of having arbitrarily small SNR is not zero, the probability of visiting the special state $s_{p}$ is non-zero, too.

Under Lemma 1, we obtain the guarantee that the optimal throughput $\eta^{*}$ is independent of the initial state [16, Prop. 7.4.1.b] and to solve equation (32) for all s, we may use two-step policy iteration algorithm for the average reward problem [16, Ch. 7.4]. In the first step, given the policy $\pi$, we calculate the corresponding average and differential rewards, $\eta$ and $h_{\mathrm{s}}$, respectively, that is, we solve the following equation for each $s \neq s_{p}$

$$
\eta+h_{\mathbf{s}}=r(\mathbf{s}, \pi(\mathbf{s}))+\sum_{\mathbf{s}^{\prime} \in \mathcal{S}} p_{\mathbf{s}, \mathbf{s}^{\prime}}\left(\pi\left(\mathbf{s}^{\prime}\right)\right) h_{\mathbf{s}^{\prime}}
$$

where $h_{\mathrm{s}_{\mathrm{p}}}=0$. In the next step, we perform a policy improvement to update $\pi(\mathrm{s})$ as follows

$$
\pi(\mathrm{s}) \leftarrow \underset{\mathrm{a} \in \mathcal{A}(\mathbf{s})}{\operatorname{argmax}}\left[r(\mathrm{~s}, \mathrm{a})+\sum_{\mathbf{s}^{\prime} \in \mathcal{S}} p_{\mathbf{s}, \mathrm{s}^{\prime}}(\mathrm{a}) h_{\mathbf{s}^{\prime}}\right] .
$$

The steps (34) and (35) are repeated till convergence, which is guaranteed to be attained in finite 
number of iterations [16, Prop. 7.4.2].

In the numerical examples, the algorithm converges with a relatively small number of iterations $(<4)$ when we choose as initial policy $\pi[\mathrm{s}]=(1 P,-)$.

\section{Numerical Results}

In this section we compare the performance of the proposed HARQ-IR, i.e., TS and SC, to the conventional HARQ-IR in terms of attainable throughput as well as the outage probability.

The throughput of the conventional HARQ-IR can be calculated using renewal-reward theorem [9], [19] or, using our MDP formulation, by considering that only the "conventional" HARQ-IR actions for all the states $s \in \mathcal{S}_{\mathrm{NACK}}^{1 \mathrm{P}}$ are taken. That is, adopting the following policy:

$$
\pi(\mathrm{s})=(1 \mathrm{P},-)
$$

1) TS vs SC: First we investigate the encoding mode to be used, i.e., we want to find the benefit of deciding in favor of the TS or SC. To this end we first run the MDP optimization for a fixed value of $R=4$ and analyze the case when $\mathcal{A}=\mathcal{A}_{\text {mod }}^{\mathrm{TS}} \times \mathcal{A}_{\mathrm{p}}$, that is, the HARQ controller is able to choose among the modes 1P, OP or TS. These results, denoted by TS, are shown in Fig. 2. We also show therein, under the legend $\mathrm{SC}$, the results of the MDP optimization when $\mathcal{A}=\mathcal{A}_{\text {mod }}^{\mathrm{SC}} \times \mathcal{A}_{\mathrm{p}}$, i.e., the HARQ controller is to choose one of the modes $1 \mathrm{P}, 0 \mathrm{P}$ or $\mathrm{SC}$. The throughput of the conventional HARQ-IR, denoted as 1P, as well as the ergodic capacity $\bar{C}$ are also shown for comparison.

Using the conventional HARQ-IR and for the fixed $R$ (i.e., that does not change with $\overline{\operatorname{snr}}$ ), the benefit of increasing the number of allowed transmission $K$ materializes only for low SNR and thus, for small throughput values. This is why HARQ is sometimes considered valuable only for low SNR regime. As we will see in Fig. 6, similar value of throughput may be obtained decreasing $R$ and yet keeping $K$ small. Therefore, from the system-level perspective, the most valuable throughput gains are those obtained close to the nominal transmission rate $R$, where we see that the multi-packet HARQ provides significant advantage over the conventional HARQ.

As a reference we consider the throughput $\eta=0.9 R$ (shown by the horizontal dashed line in Fig. 2). An important observation —already made in [25] —is that the conventional HARQ presents a large SNR gap to the ergodic capacity $\bar{C}$ when the transmission rate $R$ is fixed. For instance, the gap of approximately $8.5 \mathrm{~dB}$ can be observed in Fig. 2. This puts in the 
perspective the often evoked property of HARQ-IR, which says that the throughput of HARQIR can attain the ergodic capacity with infinite number of transmissions. While this is, indeed, true, this condition materializes for the throughput $\eta$ much smaller than $R$ or for a very small SNR. Alternatively, to reach the ergodic limit having the SNR fixed, a very large rate $R$ is needed.

From that point of view, the proposed multi-packet transmission mode seems to reduce efficiently the gap. In fact, when $R=4$ bits/channel use and $K=4$, the gap is reduced by more than $60 \%$. A multi-packet HARQ with $K=2$ can easily present a gain above $3 \mathrm{~dB}$ compared to the conventional HARQ with larger $K$. When $K=3$, the gains are around $6 \mathrm{~dB}$; they increase negligible for $K=4$.

We also note that, even if the difference is relatively small (less than $1 \mathrm{~dB}$ ), the SC always outperforms the TS. To obtain insight into the relevance of the encoding modes, considering $\mathcal{A}=\{1 \mathrm{P}, 0 \mathrm{P}, \mathrm{TS}, \mathrm{SC}\}$ and $K=2$ and defining the following probabilities conditioned on the retransmission being needed (i.e., on $\mathrm{s} \in \mathcal{S}_{\mathrm{ACK}, \mathrm{NACK}}$ )

$$
\begin{aligned}
P_{\mathrm{lP}, 2} & \triangleq \operatorname{Pr}\left\{\mathrm{m}[n]=1 \mathrm{P} \mid \mathrm{s} \in \mathcal{S}_{\mathrm{ACK}, \mathrm{NACK}}\right\}, \\
P_{\mathrm{SC}} & \triangleq \operatorname{Pr}\left\{\mathrm{m}[n]=\mathrm{SC} \mid \mathrm{s} \in \mathcal{S}_{\mathrm{ACK}, \mathrm{NACK}}\right\}, \\
P_{\mathrm{TS}} & \triangleq \operatorname{Pr}\left\{\mathrm{m}[n]=\mathrm{TS} \mid \mathrm{s} \in \mathcal{S}_{\mathrm{ACK}, \mathrm{NACK}}\right\}, \\
P_{\mathrm{Drop}} & \triangleq \operatorname{Pr}\left\{\mathrm{m}[n]=0 \mathrm{P} \mid \mathrm{s} \in \mathcal{S}_{\mathrm{ACK}, \mathrm{NACK}}\right\}, \\
P_{\mathrm{ACK}, 1} & \triangleq \operatorname{Pr}\left\{\mathrm{s} \in \mathcal{S}_{\mathrm{ACK}, \mathrm{ACK}}\right\},
\end{aligned}
$$

where $P_{1 \mathrm{P}, 2}$ is the probability of choosing one-packet retransmission, $P_{\mathrm{SC}}$ is the probability of choosing SC encoding, $P_{\mathrm{TS}}$ is the probability of choosing TS encoding, $P_{\mathrm{Drop}}$ is the probability of "dropping" the packet without retransmission and $P_{\mathrm{ACK}, 1}$ is the probability that a packet is decoded after the first transmission.

Fig. 3 shows the above-defined probability as a function of $\overline{\operatorname{snr}}$ from which the following observations can be made

- Below a threshold $(\overline{\mathrm{snr}}=18 \mathrm{~dB})$, it is more profitable from the throughput point of view to drop the packet, which corresponds to the action $\mathrm{a}=(0 \mathrm{P},-)$.

- The one-packet encoding dominates the multi-packet encoding for $\overline{\mathrm{snr}}<10 \mathrm{~dB}$, which 


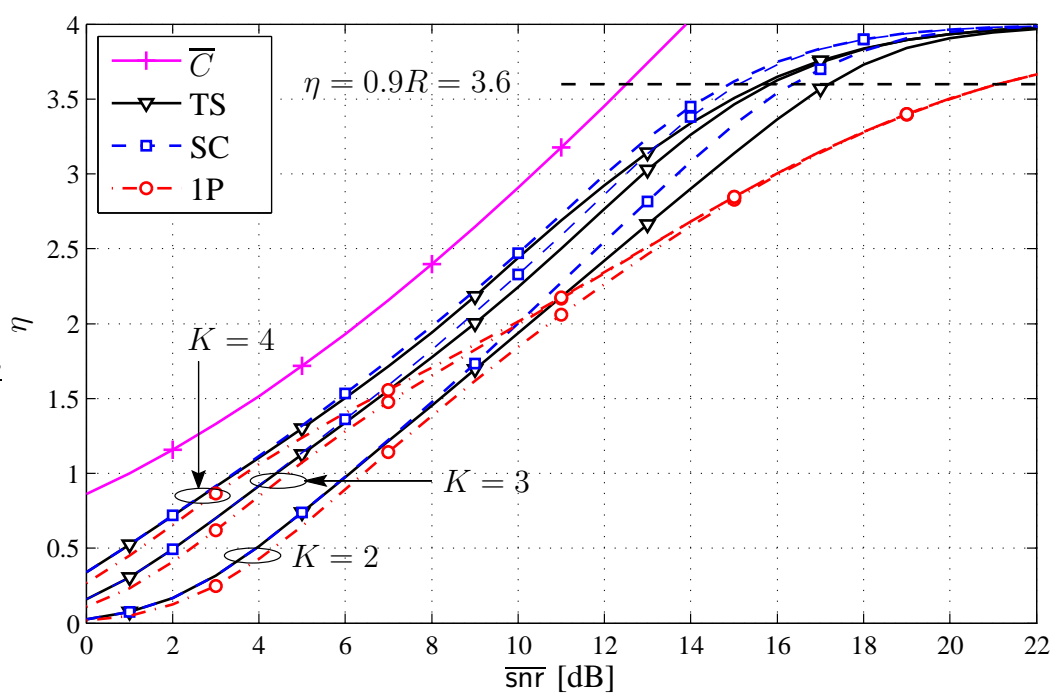

Fig. 2: Throughput of the proposed multi-packet HARQ TS and SC with $T_{\mathrm{p}}=32$ are compared to the conventional HARQ $1 \mathrm{P}$ and the ergodic capacity $\bar{C}$ when $R=4 \mathrm{bits} / \mathrm{channel}$ use and $K=2,3$ and 4 .

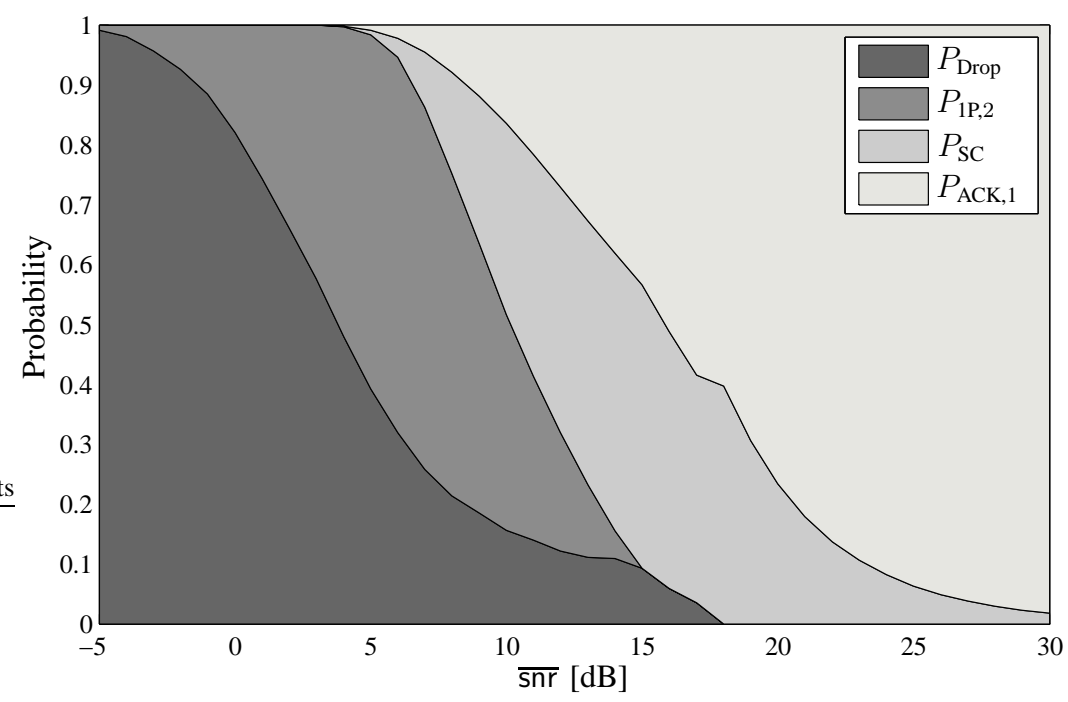

Fig. 3: Probabilities defined in (37)-(41) with $R=4$ bits/channel use and $K=2$.

explains the throughput results are similar for the conventional and the proposed multipacket HARQ.

- The multi-packet SC transmission is likely to be used for $10 \mathrm{~dB}<\overline{\mathrm{snr}}<25 \mathrm{~dB}$; this region of SNR corresponds also to the throughput of the multi-packet HARQ (SC) being 
significantly larger than the throughput of the conventional HARQ (1P).

- When SC mode is available, the time-sharing mode is never used.

- Asymptoticaly, i.e., increasing the SNR, we increase the probability of successful decoding in the first transmission, thus all HARQ modes will offer a similar throughput for large SNR.

The price to pay for the larger throughput is the increase in the outage as we illustrate in Fig. 4. While outage considerations were absent from our discussion, we note that it is also possible to design the policies which take into account the constraints on the outage, however, we leave this issue beyond the scope of our work.

In Fig. 5 we show an example of the optimal value of the parameter $p$ as a function of the AMI $I_{\ell, 1}$, i.e., when the optimal actions are $\mathrm{a}(\mathrm{s})=(\mathrm{TS}, p)$ or $\mathrm{a}(\mathrm{s})=(\mathrm{SC}, p)$, and when $\boldsymbol{k}^{\mathrm{s}}=(1,0)$. The intuition behind such results is clear: for larger $I_{\ell, 1}$, i.e., when the first transmission results are close to being decoded (this happens when $I_{\ell, 1}>R=4$, the power (for SC) or the time (for TS) fractions attributed to the retransmission decrease.

2) Comparison for different $R$ : We show the results of the throughput for different values $R$ in Fig. 6; the results shown for $R=4$ are the same as those we already presented in Fig. 2. As we can see, the gains of the SC over the TS are less pronounced for smaller values of $R$ and $\overline{\operatorname{snr}}$.

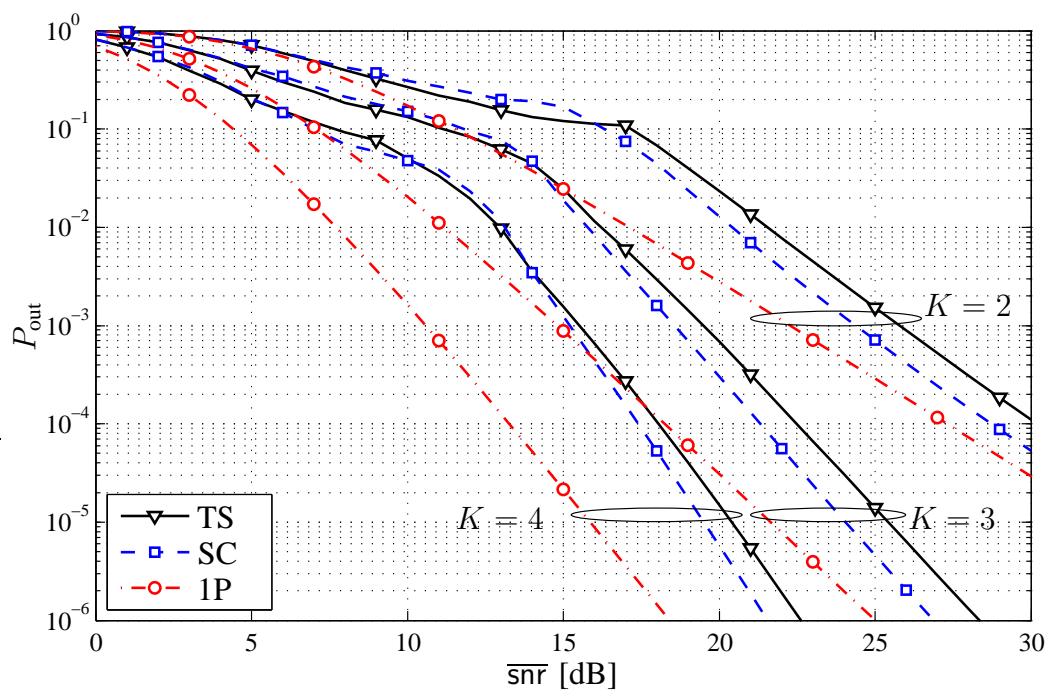

Fig. 4: Outage corresponding to the one-packet and multiple-packet HARQ when $R=4$ bits/channel use and $T_{\mathrm{p}}=32$. 


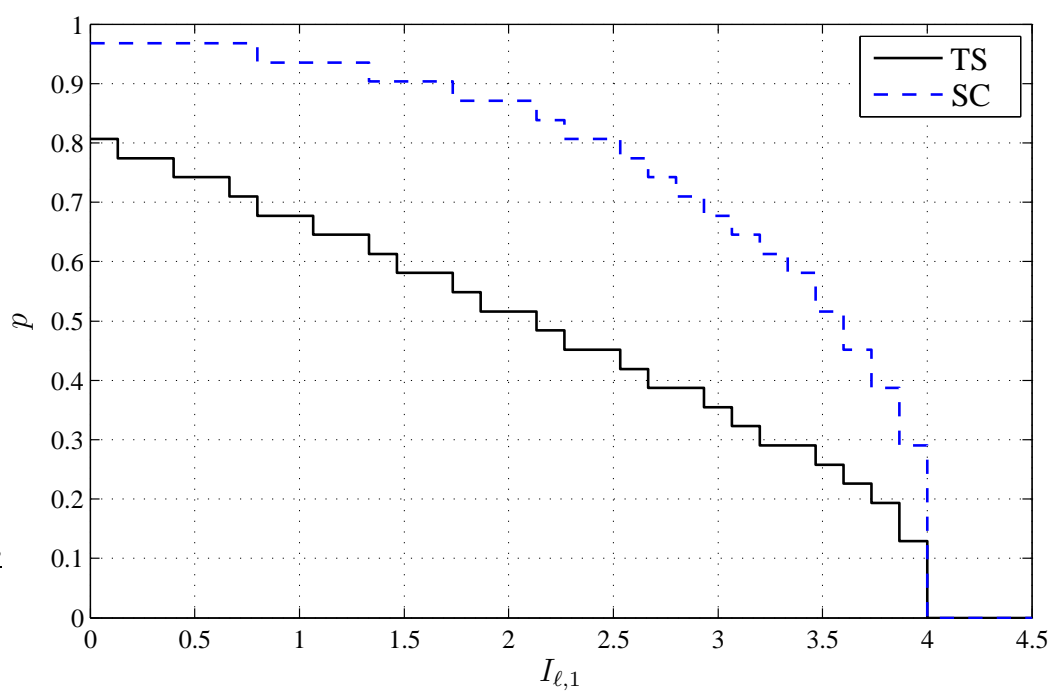

Fig. 5: An example of the optimal parameter $p$ obtained for TS and SC when $R=4$ bits/channel use, $\overline{\mathrm{snr}}=16 \mathrm{~dB}, T_{\mathrm{p}}=32$ and $K=4$.

This is reminiscence of the similar behaviour of the conventional SC broadcast transmission, where the gains with respect to the TS appear also in high SNR.

The main conclusion is that the multi-packet HARQ provides an important increase of the throughput in the zone of interest (that is, for throughput values close to the nominal transmission rate $R$ ).

3) A Note on Discretization: To provide an insight into the discretization effects, we show Fig. 7. We emphasize here that we took a sufficiently large $T_{\mathcal{I}}$ to accurately calculate the throughput ( $T_{\mathcal{I}}=32$ in the numerical examples). Thus, discretization effects are almost entirely captured by $T_{\mathrm{p}}$. We note that TS and SC present notable gain compared to $1 \mathrm{P}$ with only $T_{\mathrm{p}}=4$. The results do not change significantly for $T_{\mathrm{p}} \geq 16$. For a given $T_{\mathrm{p}}$, performance may be improved if we considered non uniform quantization, specially in the case of SC; the issue of finding the optimal quantization is, however, out of scope of this work.

\section{ONE-BIT FEEDBACK SCENARIO}

In this section, we consider the scenario where only the conventional 1-bit feedback ACK/NACK is available at the transmitter. In this case, the state space $\mathcal{S}=\mathcal{K} \times \mathcal{I}^{2}$ is partially observable: the random variable $\mathcal{I}^{2}$ is non-observable while $\mathcal{K}$ is fully observable from transmitter by using 


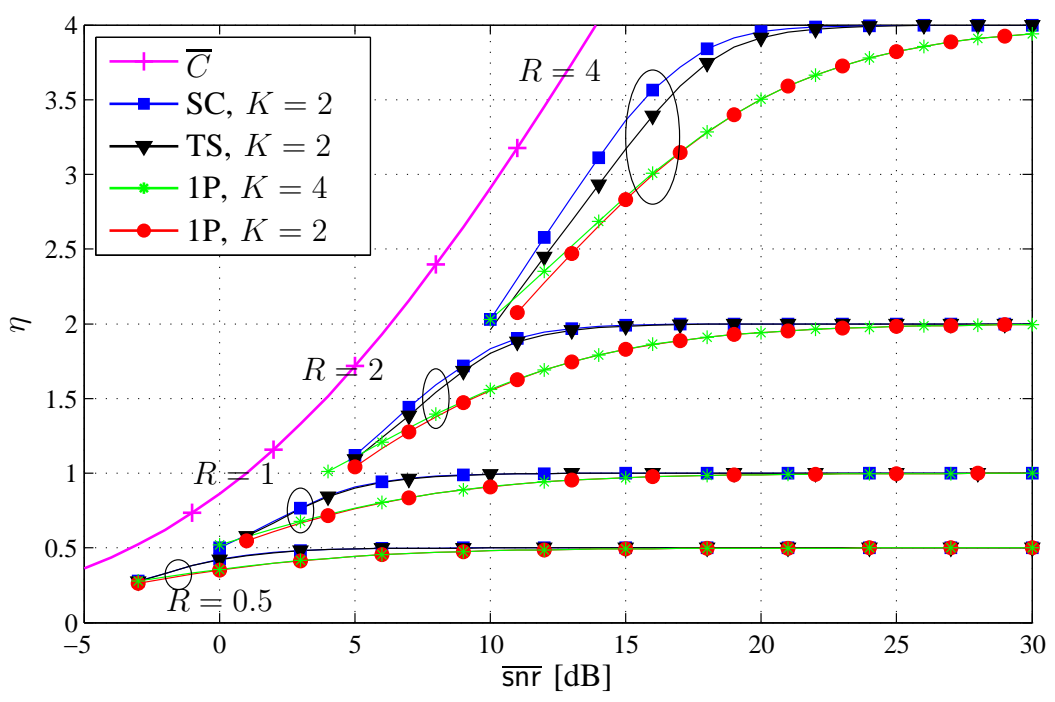

Fig. 6: Throughput obtained for various values of $R$ bits/channel use, where for clarity, the results are shown only for $\eta>R / 2$.

only the received ACK/NACK bits. This situation is known in the literature as Partial State Information Markov Decision Process (PSI-MDP) [26].

In the PSI-MDP context, the HARQ controller decides to take actions $\mathrm{a}[n]$ on the basis of observable history defined as:

$$
\mathcal{O}[n]=(\mathrm{s}[0], \mathrm{a}[0], \mathcal{M}[1], \boldsymbol{k}[1], \mathrm{a}[1], \cdots, \mathrm{a}[n-1], \mathcal{M}[n], \boldsymbol{k}[n])
$$

where the initial state $s[0]$ and the corresponding action $a[0]$ are assumed to be known. At the transmitter, the $k_{\ell}$ are updated using the observable feedback messages $\mathcal{M}[n]$; the past actions a are also perfectly known to the transmitter. In general, not the entire history is useful to the controller but only the parts related to the packets under transmission at time $n$, i.e., HoL and HoL-next packet.

The standard procedure to solve PSI-MDP problem consists in finding the equivalent perfect state $\operatorname{MDP}$ problem $\left(\mathcal{Z}, \mathcal{A}, \mathcal{W}, Q^{\prime}, r^{\prime}\right)$ [16]. The state space $\mathcal{Z}$ is $\mathcal{K} \times \mathcal{P}\left(\mathcal{I}^{2}\right)$, with $\mathcal{P}\left(\mathcal{I}^{2}\right)$, called the belief states, is the space of all probability measures on $\mathcal{I}^{2}$. The definition of the action space $\mathcal{A}$ and the disturbance space $\mathcal{W}$ are the same as in the sec. III.

Let $\mathrm{z} \in \mathcal{Z}$ be defined as the couple $(k, b)$ where $b(x)=p_{\mathbf{I}}(x / \mathcal{O}[n])$ is the a posteriori distribution of $\mathbf{I}=\left(I_{\ell, k_{\ell}}, I_{\ell+1, k_{\ell+1}}\right)$ given an observable history $\mathcal{O}$. The statistical evolution of 


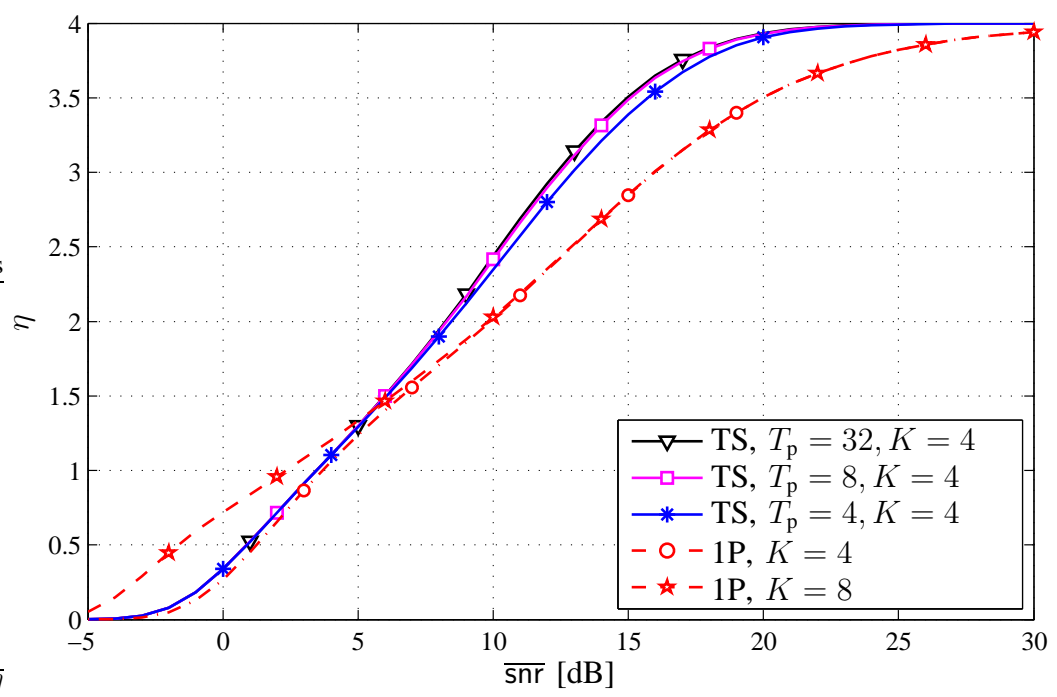

(a)

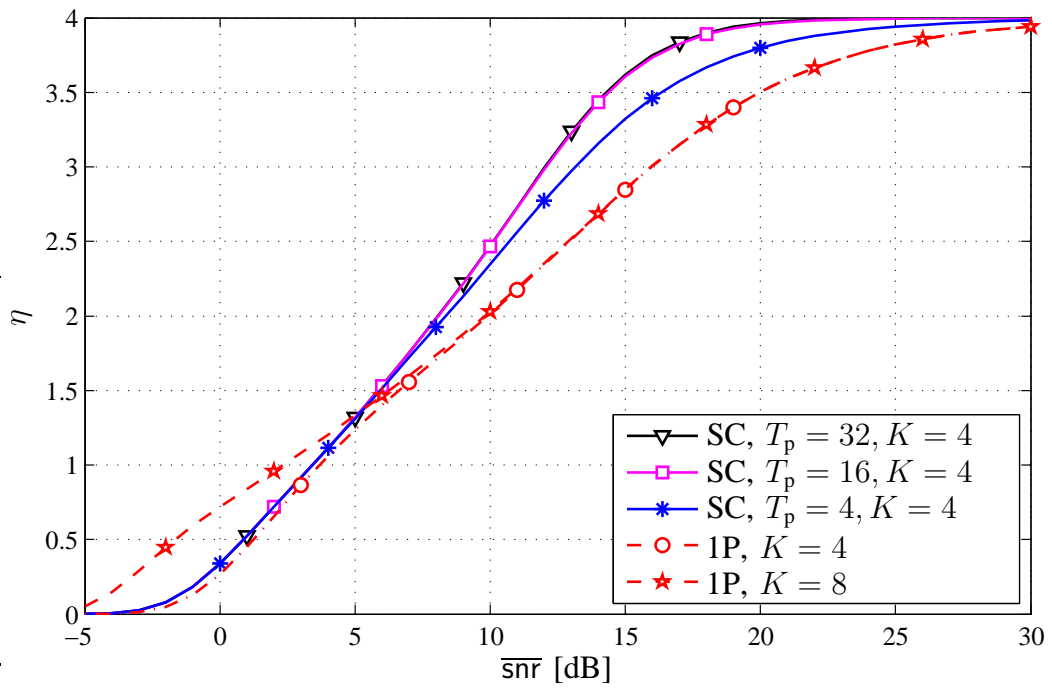

(b)

Fig. 7: Throughput of the proposed multi-packet in the case of (a) HARQ with TS and (b) HARQ with SC. Different values of $T_{\mathrm{p}}$ are considered and $R=4$ bits/channel use. The throughput of the conventional HARQ $1 \mathrm{P}$ is shown for comparison.

the system is captured by the transition law $Q^{\prime}: \mathcal{Z} \times \mathcal{A} \mapsto \Pi(\mathcal{Z})$ where $\Pi(\mathcal{Z})$ presents the set of discrete probability distribution over $\mathcal{Z}$. The expected reward $r^{\prime}(z, a)$ for taking the action a in the state $z$ is then given by:

$$
r^{\prime}(\mathrm{z}, \mathrm{a})=\int_{\mathcal{I}^{2}} \hat{r}(\mathrm{z}, \mathrm{a}, x) b(x) \mathrm{d} x
$$




$$
b(x)=\left\{\begin{array}{lc}
\frac{\log (2) \cdot 2^{\frac{x}{1-p}} \cdot p_{\mathrm{snr}}\left(2^{\frac{x}{1-p}}-1\right)}{(1-p) \cdot F_{\mathrm{snr}}\left(2^{\frac{R}{1-p}}-1\right)} \cdot \mathbb{I}(x \leq R) & \text { if } \mathcal{M}[n] \in\{(\mathrm{ACK}, \mathrm{NACK}),(\mathrm{NACK}, \mathrm{NACK})\}, \\
\frac{\log (2) \cdot(1-p) \cdot 2^{x}}{\left(1-p \cdot 2^{x}\right)^{2} \cdot \operatorname{Pr}\{\mathrm{NACK}\}} \cdot p_{\mathrm{snr}}\left(\frac{2^{x}-1}{1-p \cdot 2^{x}}\right) \cdot \mathbb{I}\left(p<2^{-x}\right) & \text { if } \quad \mathcal{M}[n]=(\mathrm{NACK}, \mathrm{NACK}), \boldsymbol{k}[n]=(2,1), \\
\frac{\log (2) \cdot 2^{x}}{(1-p) \cdot F_{\mathrm{snr}}\left(\frac{2^{R}-1}{1-p}\right)} \cdot p_{\mathrm{snr}}\left(\frac{2^{x}-1}{1-p}\right) \cdot \mathbb{I}(x \leq R) & \mathrm{m}[n]=\mathrm{SC}, \mathrm{m}[n+1] \in\{\mathrm{SC}, 1 \mathrm{P}\} \\
\frac{\log (2) \cdot 2^{x} \cdot p_{\mathrm{snr}}\left(2^{x}-1\right)}{F_{\mathrm{snr}}\left(2^{R}-1\right)} \cdot \mathbb{I}(x \leq R) & \text { if } \quad \mathcal{M}[n]=(\mathrm{ACK}, \mathrm{NACK}), \boldsymbol{k}[n]=(2,1), \\
& \mathrm{m}[n]=\mathrm{SC}, \mathrm{m}[n+1] \in\{\mathrm{SC}, 1 \mathrm{P}\}
\end{array}\right.
$$

The main challenge in solving the PSI-MDP problem is the characterization of the space of the belief states $\mathcal{P}\left(\mathcal{I}^{2}\right)$, i.e., the space of functions $b(x)$.

\section{A. Case of maximum two allowed transmission}

When $K=2$, we only need to track the value $I_{\ell, 1}$ or $I_{\ell+1,1}$ when a NACK message is received, i.e., the AMI after the first transmission of the HoL, or HoL-next packet. Thus, the belief states $b(x)$ need to be defined over only one dimension and will be parametrized by the set of possible actions. In other words, we define the state space using the actions $\mathrm{a}[n-1]$ and the feedback message $\mathcal{M}[n]$. The closed form expression are given in (45) where $\mathbb{I}(x)=1$ if $x$ is true, and 0 otherwise, $F_{\text {snr }}(x)=1-\exp (-x / \overline{\mathrm{snr}})$ is the of snr' cumulative density function (CDF) and $\operatorname{Pr}\{$ NACK $\}$ is the probability of not decoding the superposed HoL-next packet given that the HoL packet was also not decoded:

$$
\operatorname{Pr}\{\mathrm{NACK}\}=\left\{\begin{array}{ll}
F_{\mathrm{snr}}\left(\frac{2^{R}-1}{1-p \cdot 2^{R}}\right), & \text { if } p<2^{-R} \\
1, & \text { otherwise }
\end{array} .\right.
$$




\section{B. General case}

When $K>2$, the belief states $b(x)$ are defined over two dimensions and cannot be derived in closed form.

When the belief states are defined over one dimension, an approximation method was proposed in [27], which projects the beliefs on the parametrized set of functions. For example, $\operatorname{Beta}\left(\theta_{1}, \theta_{2}\right)$ function was used in [27] to parametrize the AMI $I_{\ell, k_{\ell}}$ ( [27] considered one-packet HARQ). In

our case, the observations $I_{\ell, k_{\ell}}$ and $I_{\ell+1, k_{\ell+1}}$ are dependant and we would need a projection of the joint PDF of these two variables on the space of two-dimensional functions. This approach is thus tedious and to overcome this difficulty, we assume that if a retransmission is needed, the controller will always adopt the unique-action policy:

$$
\pi(\mathrm{s})=\hat{\mathrm{a}} .
$$

Formally, the objective is to solve:

$$
\tilde{\eta}=\max _{\hat{\mathrm{a}} \in \mathcal{A}} \eta(\pi)
$$

and an exhaustive research over the one-dimensional space of allowed actions $\mathcal{A}$ is sufficient to determine the suboptimal action.

\section{Numerical Results}

Fig. 8 compare the performance of the proposed multi-packet HARQ protocols. When the whole history $\mathcal{O}$ is used, the results are denoted by $\tilde{\eta}$ (for $K=2$ ), while $\hat{\eta}$ presents the simplified policy in (46).

We observe that

- For TS, the simplified unique-action protocol defined in (46) yields practically the same throughput as the one based on the complete parametrization of the state-space (for $K=2$ ). We thus conjecture that the same results will be obtained for $K>2$.

- As expected, using one-bit feedback introduces the penalty with respect to multi-bit feedback but still, using TS, the gains over the conventional HARQ are notable, varying from $2.5 \mathrm{~dB}$ (for $K=2$ ) to $4 \mathrm{~dB}$ (for $K=4$ ).

- For SC, the simplified unique-action protocol yields the same results as the one-packet 


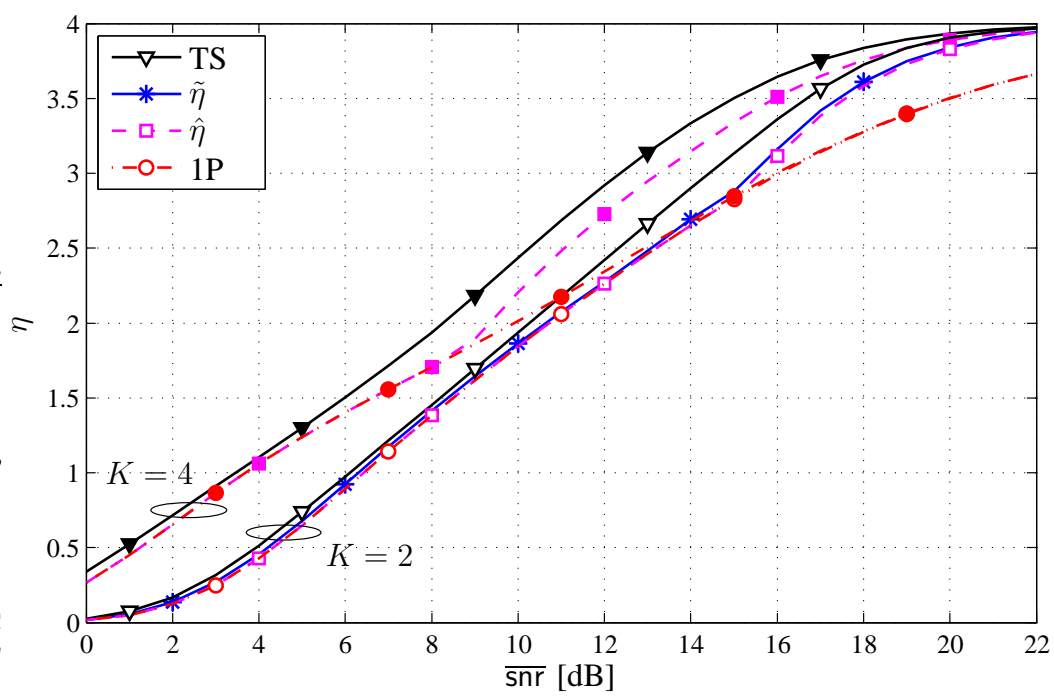

(a)

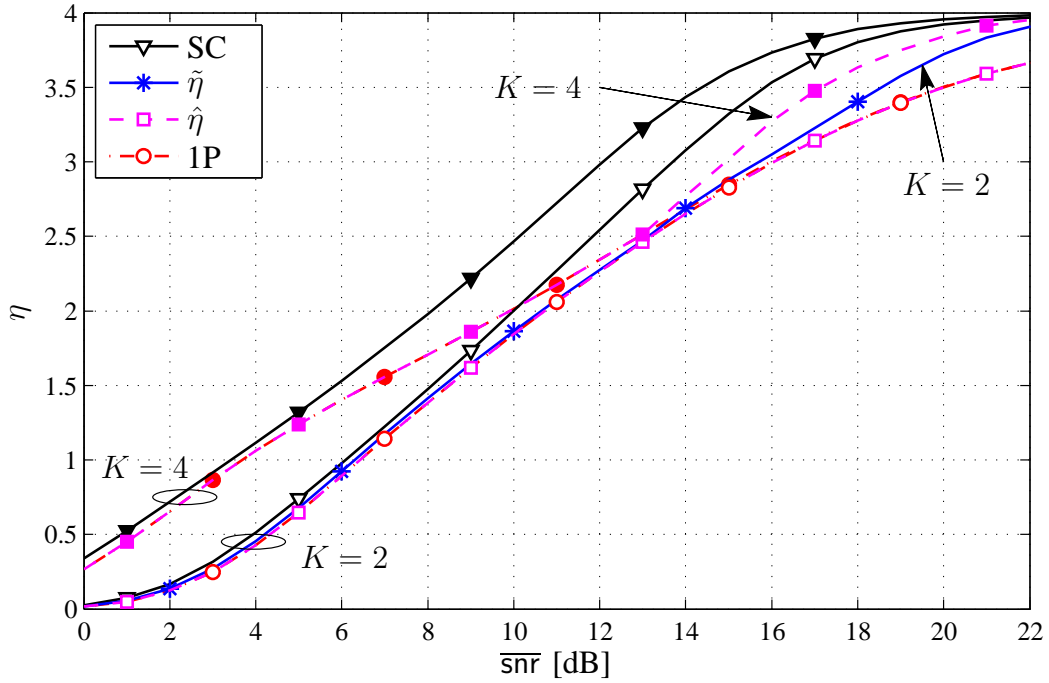

(b)

Fig. 8: Throughput of the multi-packet HARQ in the case of (a) $\mathcal{A}=\mathcal{A}_{\text {mod }}^{\mathrm{TS}} \times \mathcal{A}_{\mathrm{p}}$ and (b) $\mathcal{A}=\mathcal{A}_{\text {mod }}^{\mathrm{SC}} \times \mathcal{A}_{\mathrm{p}}$; the multi-bit feedback (TS), one-bit feedback $(\tilde{\eta})$, unique-action one-bit feedback $(\hat{\eta})$ and conventional one-packet HARQ (1P). $T_{\mathrm{p}}=16$ and $R=4$ bits/channel use.

transmission for $K=2$; this is not entirely surprising as we already observed that the SC is very sensitive to the discretization of the parameter space $A_{\mathrm{p}}$ in Fig. 7 (b). For $K=4$, we obtain an appreciable gain of $3 \mathrm{~dB}$.

- When the complete parametrization of the state-space is used (for $K=2$ ), the gains of SC over the conventional HARQ are around $1.5 \mathrm{~dB}$. 


\section{CONCLUSIONS}

In this work we proposed and analyzed the so-called multi-packet HARQ, where various packets are simultaneously transmitted within the same block. We consider adjusting the joint encoding modes depending on the state of the receiver in the past transmissions and, formulating the problem as partial or full state information Markov decision process, we optimize the encoding modes and parameters of the HARQ. Our results indicate that the joint encoding yields gains of various $\mathrm{dB}$ over the conventional HARQ even in the simple case of one-bit feedback and HARQ truncated to $K=2$ transmission. These gains can be increased by $1-2 \mathrm{~dB}$ by adding a few $(3-4)$ additional feedback bits.

We also observed that the gains of the SC with respect to the TS are relatively small in the case of full observable state; one-bit feedback, however, it removes the advantage of the SC. Thus, in the point-to-point HARQ, the TS, being simpler to implement, should be preferred over SC.

\section{APPENDIX A}

We aim at determining the expression of $p_{\mathrm{s}, \mathrm{s}^{\prime}}$ when $K=2$. In this case, the set of possible values of $\boldsymbol{k}=\left(k_{\ell}, k_{\ell+1}\right)$ is $\mathcal{K}=\{(1,0),(2,1),(2,0)\}$. One possible illustration of the state space $\mathcal{S}$ is given in Fig. 9. For each possible value of $\boldsymbol{k}$, the vertical line presents the possible values of $I_{\ell, k_{\ell}}$ while the horizontal line presents $I_{\ell+1, k_{\ell+1}}$. When only the HoL packet is transmitted, i.e., $I_{\ell+1, k_{\ell+1}}=0$, the horizontal line is irrelevant. For convenience we refer to the discretization interval corresponding to a state $s$ as $\mathcal{I}^{\mathrm{s}}$.

The state $s$ in the figure corresponds to parameter $\boldsymbol{k}^{\mathbf{s}}=(1,0)$ and $\boldsymbol{I}^{\mathbf{s}}=\left(I_{\ell, 1}^{\mathbf{s}}, 0\right)$. The transition from the state $\mathrm{s}$ at time $n$ to the state $\mathrm{s}^{\prime}$ at time $n+1$ depends on the action $\mathrm{a}[n]=(\mathrm{m}[n], p[n])$ and the SNR. For example three situations can occur regarding the value of $\mathrm{m}[n]$. Namely:

- If $\mathrm{m}[n]=1 \mathrm{P}$ the system moves to $\boldsymbol{k}^{\mathbf{s}^{\prime}}=(2,0)$. This transition, represented by a solid arrow, has the probability $p_{\mathrm{s}, \mathrm{s}^{\prime}}(\mathrm{a})=\operatorname{Pr}\left\{I_{\ell, 1}^{\mathrm{s}}+C(\mathrm{snr}) \in \mathcal{I}_{\ell, 2}^{\mathbf{s}^{\prime}}\right\}$.

- If $\mathrm{m}[n]=0 \mathrm{P}$ the HARQ controller increments the HoL according to (13) and the system moves to $\boldsymbol{k}^{\mathbf{s}^{\prime}}=(1,0)$, which is presented by the doted arrow. In this case, $p_{\mathrm{s}, \mathrm{s}^{\prime}}(\mathrm{a})=$ $\operatorname{Pr}\left\{C(\right.$ snr $\left.) \in \mathcal{I}_{\ell, 1}^{\mathrm{s}^{\prime}}\right\}$.

- If $\mathrm{m}[n]=\mathrm{TS}$ the multi packet scenario is adopted and the counters are updated to $\boldsymbol{k}^{\mathbf{s}^{\prime}}=$ $(2,1)$. This situation is depicted by the dashed arrow and $p_{\mathbf{s}, s^{\prime}}(\mathbf{a})=\operatorname{Pr}\left\{I_{\ell, 1}^{\mathrm{s}}+p C(\mathrm{snr}) \in\right.$ 


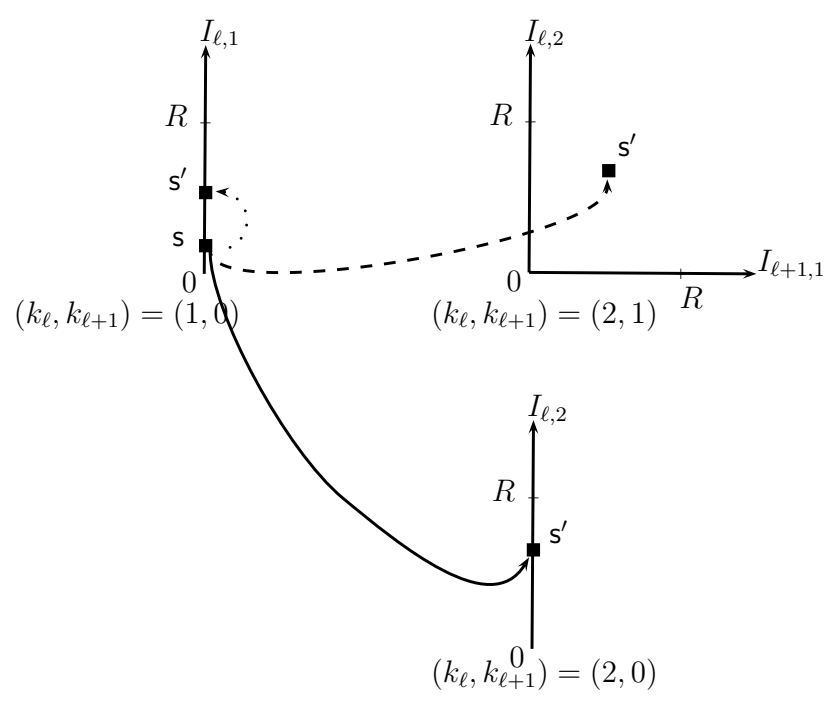

Fig. 9: State space $\mathcal{S}$ representation when $K=2$

$\left.\mathcal{I}_{\ell, 2}^{\mathrm{s}^{\prime}} \cap(1-p) C(\mathrm{snr}) \in \mathcal{I}_{\ell+1,1}^{\mathrm{s}^{\prime}}\right\}$

\section{REFERENCES}

[1] J.-F. Cheng, Y.-P. Wang, and S. Parkvall, "Adaptive incremental redundancy," in IEEE Veh. Tech. Conf., Orlando, Florida, USA, Oct. 2003, pp. 737-741.

[2] E. Uhlemann, L. K. Rasmussen, A. Grant, and P.-A. Wiberg, "Optimal incremental-redundancy strategy for type-II hybrid ARQ," in Proc. IEEE International Symposium on Information Theory, 2003, p. 448.

[3] E. Visotsky, Y. Sun, V. Tripathi, M. Honig, and R. Peterson, "Reliability-based incremental redundancy with convolutional codes,” IEEE Trans. Commun., vol. 53, no. 6, pp. 987 - 997, Jun. 2005.

[4] S. Pfletschinger and M. Navarro, "Adaptive HARQ for imperfect channel knowledge," in 2010 International ITG Conference on Source and Channel Coding (SCC), Jan. 2010, pp. 1 -6.

[5] L. Szczecinski, S. R. Khosravirad, P. Duhamel, and M. Rahman, "Rate allocation and adaptation for incremental redundancy truncated HARQ," IEEE Trans. Commun., vol. 61, no. 6, pp. 2580-2590, June 2013.

[6] R. Liu, P. Spasojevic, and E. Soljanin, "On the role of puncturing in hybrid ARQ schemes," in Proc. IEEE International Symposium on Information Theory, Jun. 2003, p. 449.

[7] S. M. Kim, W. Choi, T. W. Ban, and D. K. Sung, "Optimal rate adaptation for hybrid ARQ in time-correlated Rayleigh fading channels," IEEE Trans. Wireless Commun., vol. 10, no. 3, pp. 968 -979, Mar. 2011.

[8] K. Nguyen, L. Rasmussen, A. Guillen i Fabregas, and N. Letzepis, "MIMO ARQ with multibit feedback: Outage analysis," IEEE Trans. Inf. Theory, vol. 58, no. 2, pp. 765 - 779, Feb. 2012.

[9] X. Gan and Z. Cao, "Instantaneous packet information based on-demand adaptive retransmission for HARQ," Military Comm. Conf. 2010, MILCOM'10, pp. 1496-1501, Oct 2010. 
[10] X. Wang, Q. Liu, and G. Giannakis, "Analyzing and optimizing adaptive modulation coding jointly with ARQ for QoSguaranteed traffic,” IEEE Trans. Veh. Technol., vol. 56, no. 2, pp. 710-720, Mar. 2007.

[11] S. Shamai and A. Steiner, "A broadcast approach for a single-user slowly fading MIMO channel," IEEE Trans. Inf. Theory, vol. 49, no. 10, pp. 2617-2635, Oct. 2003.

[12] R. Zhang and L. Hanzo, "Superposition-coding-aided multiplexed hybrid ARQ scheme for improved end-to-end transmission efficiency," IEEE Trans. Veh. Technol., vol. 58, no. 8, pp. 4681 -4686, Oct. 2009.

[13] F. Takahashi and K. Higuchi, "HARQ for predetermined-rate multicast channel," Vehicular Technology Conference (VTC 2010-Spring), pp. 1-5, May 2010.

[14] T. V. K. Chaitanya and E. G. Larsson, "Superposition modulation based symmetric relaying with hybrid ARQ: Analysis and optimization,” IEEE Trans. Veh. Technol., vol. 60, no. 8, pp. 3667-3683, Oct. 2011.

[15] S. Verdu and S. Shamai, "Variable-rate channel capacity," IEEE Trans. Inf. Theory, vol. 56, no. 6, pp. 2651-2667, June 2010 .

[16] D. P. Bertsekas, Dynamic Programming and Optimal Control, 3rd ed. Athena Scientific, 2005, vol. 1.

[17] A. Karmokar, D. Djonin, and V. Bhargava, "Delay-aware power adaptation for incremental redundancy hybrid ARQ over fading channels with memory," IEEE International Conference on Communications, ICC'06, vol. 9, pp. 4315-4320, Jun 2006.

[18] D. Djonin, A. Karmokar, and V. Bhargava, "Joint rate and power adaptation for type-I hybrid ARQ systems over correlated fading channels under different buffer-cost constraints," IEEE Trans. Veh. Technol., vol. 57, no. 1, pp. 421 -435, Jan. 2008.

[19] G. Caire and D. Tuninetti, "The throughput of hybrid-ARQ protocols for the Gaussian collision channel," IEEE Trans. Inf. Theory, vol. 47, no. 5, pp. 1971-1988, Jul. 2001.

[20] P. Wu and N. Jindal, "Performance of hybrid-ARQ in block-fading channels: A fixed outage probability analysis," IEEE Trans. Commun., vol. 58, no. 4, pp. 1129 -1141, Apr. 2010.

[21] G. Benelli, “An ARQ scheme with memory and soft error detectors," IEEE Trans. on Commun., vol. 33, no. 3, pp. 285-288, Mar 1985.

[22] D. Chase, "Code combining- a maximum-likelihood decoding approach for combining an arbitrary number of noisy packets," IEEE Trans. Commun., vol. 33, no. 5, pp. 385-393, May 1985.

[23] J. Hagenauer, "Rate-compatible punctured convolutional codes (rcpc codes) and their applications," IEEE Trans. Commun., vol. 36, no. 4, pp. $389-400$, apr 1988.

[24] M. Jabi, L. Szczecinski, M. Benjillali, and F. Labeau, "Outage-optimal power adaptation and allocation for truncated HARQ," IEEE International Conference on Communications, ICC'14, pp. 1890-1896, June 2014.

[25] P. Larsson, L. Rasmussen, and M. Skoglund, "Throughput analysis of ARQ schemes in Gaussian block fading channels," IEEE Trans. Commun., vol. PP, no. 99, pp. 1-1, PP 2014.

[26] A. Arapostathis, V. S. Borkar, E. Fernandez-Gaucherand, M. K. Ghosh, and S. I. Marcus, "Discrete-time controlled markov processes with average cost criterion: a survey," SIAM J. Control Optim., vol. 31 (2), pp. 282-344, 1993.

[27] R. Tajan, "Mecanismes de retransmission Hybrid-ARQ en radio-cognitive.” Ph.D. dissertation, Cergy-Pontoise University, France, 2013. 